\title{
Molecular characterization of Moniliophthora roreri, causative agent of moniliasis in cocoa in three provinces of Ecuador: Los Ríos, Manabí and Santo Domingo de los Tsáchilas
}

\author{
Mollocana, D. ${ }^{a}$, Erazo, $M P{ }^{a}{ }^{a}$, Aguirre, $X{ }^{a}$, Torres, $M L^{a}{ }^{a}$ \\ ${ }^{a}$ Universidad San Francisco de Quito USFQ. Laboratorio de Biotecnología Vegetal, Colegio de \\ Ciencias Biológicas y Ambientales. EC170157, Quito, Ecuador.
}

\section{Graphical Abstract

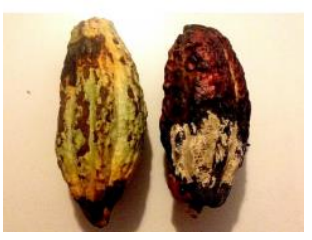 \\ Cacao cobs infected with moniliasis

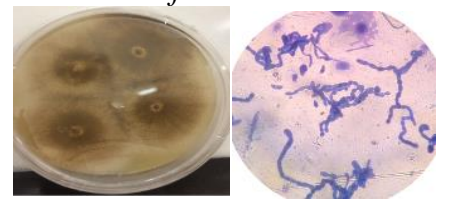

M. roreri growth. in culture medium V8 and microscopic view of the culture (1000x magnification).

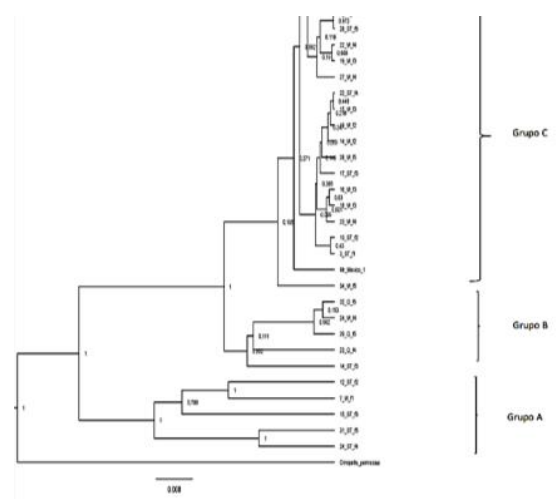

Phylogenetic Tree constructed from the M. roreri sequences of this study (75) Los Ríos (Q), Santo Domingo (SD) Manabi

(M) and other Latin American sequences obtained from GenBank.

\begin{abstract}
.
Cocoa (Theobroma cacao $L$ ) is one of the main agricultural products of Ecuador. However, it is susceptible to diseases and pests being those of fungal origin, the most common [1]. Black sheath caused by Phytophthora sp., the witch's broom caused by Moniliophthora perniciosa, and moniliasis caused by Moniliophthora roreri are the most reported conditions. Moniliasis is considered the most destructive than all diseases that affect this crop [2].

M.roreri manifests itself on the cob of the plant and presents two phases of infection: the biotrophic where it can be seen malformations in the fruit, and the necrotic where rot and death of the fruit occur [3]. The spore masses break off the fungus pseudostroma and move by different mechanisms (wind, humans, animals, and water) [4]. The climatic conditions and the amount of free spores are determinants in the life cycle of this pathogen [5]. Nevertheless, the fungus is present during year-round as a component of the air layer in growing regions, making eradication difficult. A better knowledge of the genetic diversity of this microorganism could lead to a
\end{abstract}


greater understanding of its distribution and adaptation mechanisms.

Since this disease causes great economic losses, and there have been few studies of its molecular level, the objective of this investigation was the characterization of $\underline{M}$. roreri isolates, to determinate its genetic diversity. To do this, 75 samples of the $\underline{M}$. roreri fungus from three Ecuadorian provinces of high cocoa productivity were taken. Nucleotide diversity was determined through the amplification and sequencing of the ITS1 and ITS2 ribosomal regions of each sample. It was found a low level of genetic differentiation between the samples analyzed. The results of the molecular variance analysis (AMOVA) revealed that most of the genetic variability is found within individuals (71.29\%). To study phylogenetic relationships, an MCC algorithm tree was made using the sequences of the ITS regions from the samples in this study, together with other continental sequences previously reported in public databases (42 samples in total). Interestingly, it was found after performing the phylogenetic analyzes that Ecuador could be the center of origin of $M$. roreri, with multiple reintroductions of the pathogen occurring throughout Latin America. Data should be corroborated with future research.

Keywords: Moniliophthora roreri, nucleotide diversity, phylogeny, ITS region

\section{References}

[1] End, M. J., Daymond, A. J., \& Hadley, P. (Eds.). (2014). Technical guidelines for the safe movement of cacao germplasm. Revised from the FAO/IPGRI Technical Guidelines No. 20 (Second Update, August 2014). Global Cacao Genetic Resources Network (CacaoNet), Bioversity International, Montpellier.

[2] Phillips-Mora, W., Aime, M. C., \& Wilkinson, M. J. (2007). Biodiversity and biogeography of the cacao (Theobroma cacao) pathogen Moniliophthora roreri in tropical America. Plant Pathology, 56, 911-922.

[3] Bailey, B. Evans, H. Phillips- Mora, W. Ali, Sh. Meinhardt, L. (2018). Moniliophthora roreri, causal agent of cacao Frost. Molecular Plant Pathology 19(7):1580-1594

[4] Philips Mora, W. 2003. Origin, biogeography, genetic diversity and taxonomic affinities of the cacao (Theobroma cacao L.) fungus Moniliophthora roreri (Cif.) Evans et al. as determined using 
http://sciforum.net/conference/mol2net-05

molecular, phytopathological and morpho-physiological evidence. (Doctoral Thesis). University of Reading. UK.

[5] González, A. 2014. Aislamiento y caracterización del hongo Moniliophthora roreri (monilia) en frutos de Theobroma cacao L. (Cacao) del cultivar San José del Real de la Carrera, USULUTAN (Thesis). Universidad de El Salvador. El Salvador. 